\title{
Supervised Learning-based Cancer Detection
}

\author{
Juel Sikder ${ }^{1}$, Utpol Kanti Das ${ }^{2}$, Rana Jyoti Chakma ${ }^{3}$ \\ Dept. of Computer Science and Engineering \\ Rangamati Science and Technology University \\ Rangamati, Bangladesh
}

\begin{abstract}
The segmentation, detection and extraction of the infected tumor from Magnetic Resonance Imaging (MRI) images are the key concerns for radiologists or clinical experts. But it is tedious and time consuming and its accuracy depends on their experience only. This paper suggest a new methodology segmentation, recognition, classification and detection of different types of cancer cells from both MRI and RGB (Red, Green, Blue) images are performed using supervised learning, Convolutional Neural Network (CNN) and morphological operations. In this methodology, $\mathrm{CNN}$ is used to classify cancer types and semantic segmentation to segment cancer cells. The system trained using the pixel labeled the ground truth where every image labeled as cancerous and non-cancerous. The system trained with $\mathbf{7 0 \%}$ images and validated and tested with the rest $30 \%$. Finally, the segmented cancer region is extracted and its percentage area is calculated. The research examined on the MATLAB platform on MRI and RGB images of the infected cell of BreCaHAD dataset for breast cancer, SN-AM Dataset for leukemia, Lung and Colon Cancer Histopathological Images dataset for lung cancer and Brain MRI Images for Brain Tumor Detection dataset for brain cancer.
\end{abstract}

Keywords-Semantic segmentation; CNN; brain; breast; leukemia; lung

\section{INTRODUCTION}

Muscles, bones, the liver and the lungs are forming rapidly from trillions of cells produced by the human body. Lung cancer starts its journey from the lung. Secondary type cancer spreads to the lung from another part of the body. Cancer which cannot compete against infection spreads in the bone marrow and also a cancer that happens in white blood cells is known as Leukemia. The brain tumor is a collection or a combination of unnatural tissue in the brain. MRI, computerized tomography (CT) scan and the Biopsy technique used to detect the brain tumor. MRI images are safer than CT scan images, because MRI can produce higher contrast compared with CT scan and MRI images also do not harm the body. As the MRI images produce a pure resolution of brain tissues, they are used in detection and classification techniques. The lymph vessels and blood vessels help breast cancer to propagate outside the breast.

Nowadays, it is common to see that deep learning-based segmentation is a more useful segmentation technique in the present world which introduces deep learning in every sphere of the digital world. Semantic segmentation is one of the best deep learning-based segmentation techniques that introduces the machine's knowledge to human beings. A good example is supervised learning that includes labeled the ground truth dataset of learning object categories. This outstanding supervised methodology used SegNet architecture for the segmentation purpose of cancer cells that are of different types.
Classification of objects is more useful and necessary for the recognition of testing objects like cancer cells in this study. This research used Convolutional Neural Network(CNN) for classification purposes. It takes the help of the resnet50 architecture that uses fc1000 layers for this deep classification of the testing image [1]. The authors implement an idea that turns the machine into a human brain. To test this study, different types of cancer datasets for segment and detect cancer cells of heterogeneous have been used. The system split all dataset images of the different format into different classes, categorized them into Breast, Leukemia, Lung and Brain where every category of the dataset is labeled using the pixel label named Cancerous and Non_Cancerous class. During performance as a human being, test image is classified using CNN classifier and based on the classified predict label, classified category along with its labeling are passed into the SegNet architecture to build a SegNet semantic segmentation procedure using VGG16 layers. Creating semantic segmentation procedure, a test image is segmented and segmented cancer cell is detected using morphological operations and detected cancer area is calculated. Many researchers have only worked with specific types of cancer cell detection with different methods, but in this research we focused on many types of cancer cell detection using the proposed system. In brief, we develop a system to segment, detect and classify the cancer cells more especially Brain, Breast, Leukemia, and Lung using semantic segmentation and classification using the CNN classifier which is more convenient than traditional existing method.

The structure of this research has been designed as follows. The next Section II contains a review of some recent related research works. The proposed methodology is described in brief in Section III. Results and discussion sections are described in Section IV and conclusions and future research plans are discussed in Section V.

\section{LitERATURE REVIEW}

There have been many works done to detect and analyze cancer cells. Some of the existing works are described below.

Fahad Lateef and Yassine Ruichek [2] have completed a survey. According to the shared conceptions underlying their structure, they categorized different methods among ten different classes in their survey. They also provided a brief description of the publicly available datasets. Besides, to measure their accuracy, they used an evaluation matrix. Furthermore, they paid attention to different methodologies and examined to achieve their desired performances. Finally, they ended the research by describing discrete amounts of open questions and their probable solutions. 
Alqazzaz, Salma, et al. [3] have proposed a methodology where they applied a CNN e.g. SegNet to 3D datasets for automation of the brain tumor and sub tumor portions segmentation, as well as enhancing tumor, edema and necrosis. They applied a process to further advance tumor segmentation. In this process, to produce four maximum feature maps, they integrated the four distinctly trained SegNet architecture. To encrypt fascinating info into a feature presentation, they combined both the intensity of the pixels of the primary MRI modalities and the large feature maps. To classify the MRI voxels, the combined features are taken as input and applied to a tree that can make decisions. The authors used the BraTS 2017 challenge dataset to evaluate their methodology.

Ahmed Ghoneim, Ghulam Muhammad, M. Shamim Hossain [4] have proposed a method to classify and detect Cervical cancer. They used CNN and extreme learning machines to classify. Images of the cells are passed into a CNNs frame so that deep-learned features can be extracted. Then, extracted features have been used to classify the test images. They claimed that the classifier gives $91.2 \%$ accuracy.

Konstantinos Kamnitsas, Christian Ledig, Virginia F. J. Newcombe, Joanna P. Simpson, Andrew D. Kane, David K. Menon, Daniel Rueckert, Ben Glocker [5] have applied effective multi-scale 3D CNN with complete associated CRF for segmentation of the brain lesion. They projected a double route i.e. three-dimensional $\mathrm{CNN}$ and 11-layers deep to segment brain lesions. The authors used BRATS 2015 and ISLES 2015 dataset to examine their architecture.

Almajalid, Rania, Juan Shan, Yaodong Du, and Ming Zhang [6] have modified the u-net model for breast ultrasound imaging (BUS) segmentation. They applied a post processing after the segmentation stage. The authors claimed that the method achieved $82.52 \%$ accuracy in the term of average DICE.

Al-jaboriy, Saif S., Nilam Nur Amir Sjarif, Suriayati Chuprat, and Wafaa Mustafa Abduallah [7] have introduced a machine learning-based autonomic leukocyte cell segmentation procedure. Using artificial neural networks (ANNs) and 4moment statistical features, the features were extracted from blast cells.

Kamal, Uday, Abdul Muntakim Rafi, Rakibul Hoque, Jonathan $\mathrm{Wu}$, and Md Kamrul Hasan [8] have proposed a deep learning model for the segmentation of lung tumors from CT scans called Recurrent 3D-DenseUNet. To differentiate between tumorous and non-tumorous image-slices, they applied morphological operations and selective thresholding. They claimed that the system gained an average dice score of 0.7228 .

\section{Methodology}

The proposed system divided into the following three steps:

- Classification stage.

- Semantic segmentation and detection stage.

- Calculate cancer area.

"Fig. 1" illustrates the proposed methodology.

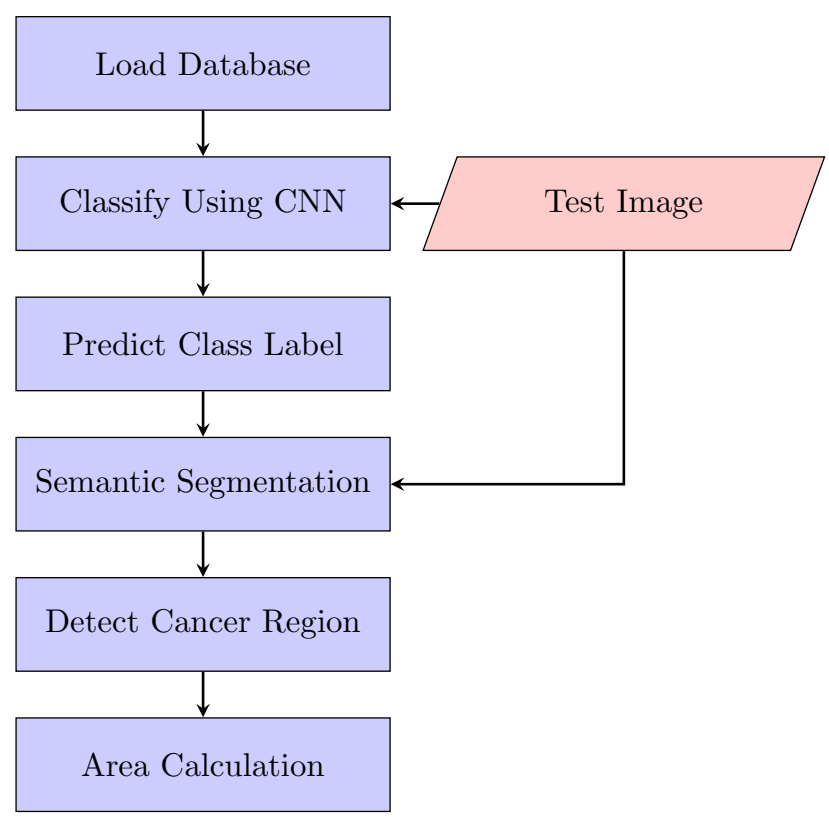

Fig. 1. Block Diagram of the Proposed System.

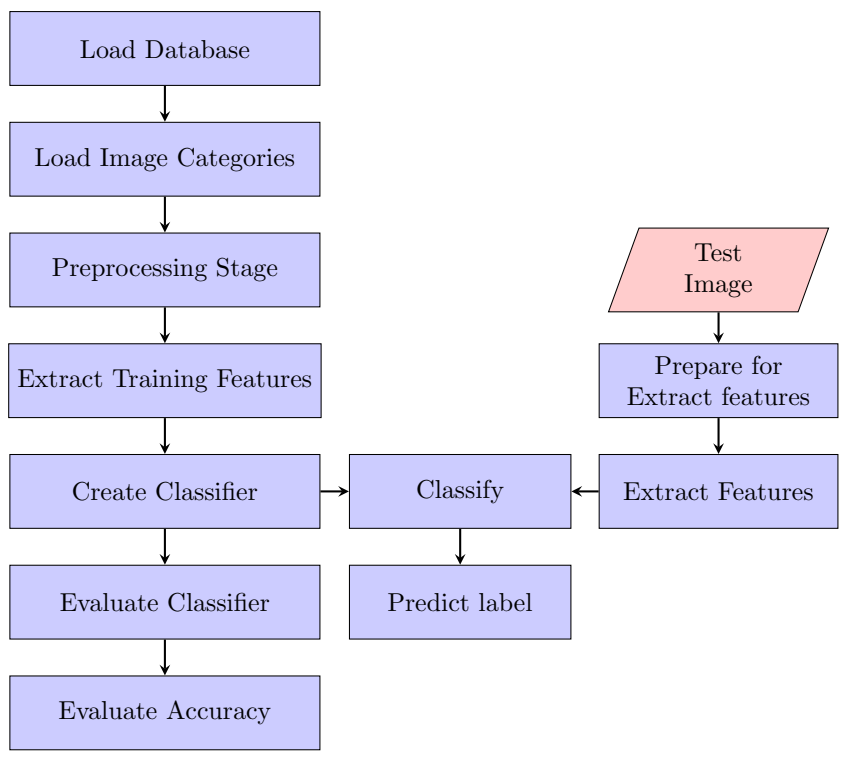

Fig. 2. Block Diagram of the CNN Classifier.

\section{A. Classification Stage}

To classify a dataset into user-defined classes, a supervised learning classification method is used [9]. It is the procedure that predicts the class of given data. The task of resembling a mapping procedure from input to output is known as classification predictive modeling. "Fig. 2" illustrates the block diagram of the CNN classifier.

For training a classifier, a pre-trained $\mathrm{CNN}$ is used as a feature extractor in the proposed system. CNNs are trained to gather a large set of varied images. From these vast collections of data, CNNs can extract important features viz. SURF, LBP 
and HOG [11].

1) Load Data Base: To test the classification approach of cancer cells, the system used Brain, Breast, Leukemia and Lung category here. The data sets loaded to the system for creating the $\mathrm{CNN}$ classifier.

2) Load image category: The system constructed an image data store (imageDatastore) function based on the following categories 'Brain', 'Breast', 'Leukemia' and 'Lung'. The system used the image data store (image Datastore) function to help manage the data. As an image data store (imageDatastore) function operates on image file locations, all the images don't load into memory.

\section{3) Preprocessing Stage:}

a) Apply CNN using ResNet-50: 'ResNet-50' net can be loaded from neural network toolbox ${ }^{\mathrm{TM}}$. The system needs to initialize 'ResNet-50' applying resnet50. The first layer shows the input dimensions. CNN required 224-by-224-by3 image size. A package of convolutional layers interspersed with rectified linear units (ReLU) and max-pooling layers exist in this architecture.

b) Prepare Training and Testing Image Sets: The system fragments the dataset into training and validation data. The system picked $60 \%$ of cancer cells for training and the remaining, $40 \%$, for the testing. It randomized the dividing process to remove any biasing. The CNN architecture processed training and testing datasets. A supervised procedure and the training data trained the model [10]. The parameters of the model are accustomed depending on the judgement results. Both the parameter estimation and variable selection can be taken by the model fitting. Using some performance metrics the accuracy of the model is evaluated using the test set. Besides the training and testing set, a validation dataset is used to validate the trained model [11]. It is a very general way to assign more than 50\% images for the training set, $25 \%$ for the test set, and the remainder for the validation set.

c) Pre-process Images For CNN: Resnet-50 uses 224by-224-3 dimension images to perform. So, the system converts grayscale images into RGB and resizes them according to the requirement of resnet50. During network training, additional data augmentation processes use that resized image. The system resized both the training and testing images.

4) Extract Training Features: To extract features, the CNN model has few layers. The features are extracted from edges and blobs at the beginning of the networks. The second convolutional layer weights are required to visualize first convolutional layer weights [12]. Then the system resized and scaled the weights for visualization. In the first layer, there are about 96 discrete groups of weights. The first convolutional layer weight is given in "Fig. 3". The activation method is used to extract the deep learning features. 'fc1000' layers are used to extract features using activation functions. Either a graphics processing unit (GPU) or a central processing unit (CPU) is used by the activation function automatically.

5) Create Classifier using extracted features: A multiclass support vector machine(SVM) classifier uses extracted features to train a classifier. The fitcecoc function is used primarily by a fast-stochastic Gradient Descent solver. To create the classifier firstly, the system collects training labels from the training

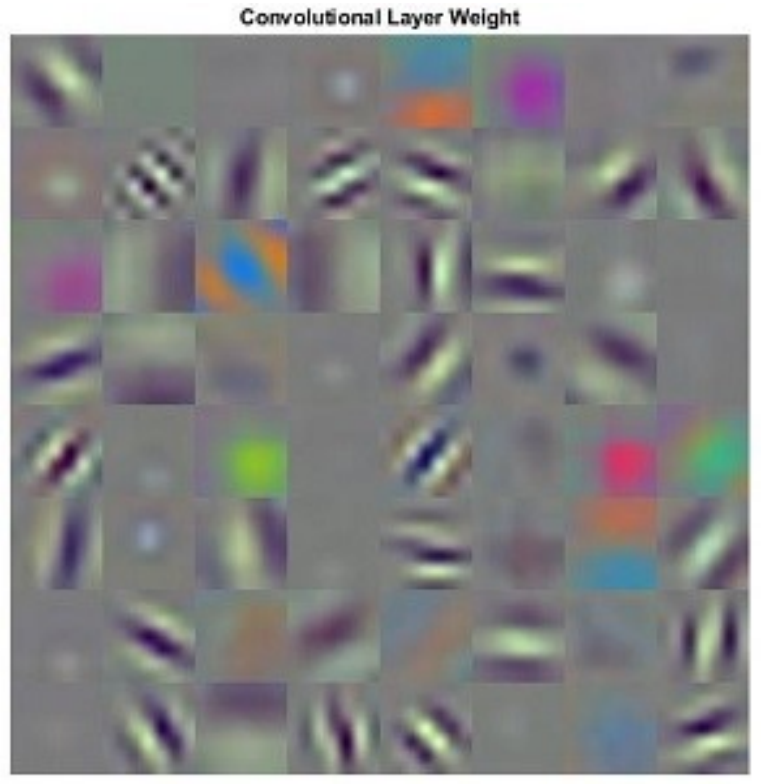

Fig. 3. First Convolutional Layer Weight.

dataset. After that multiclass SVM classifiers are trained by the system using the fastest-linear solver [13][14].

6) Evaluate Classifier: In this step, the system tests extracted features using the trained CNN classifier. To evaluate the classifier, extracted features fed to the classifier, then the system identified class labels and tabulated the outcomes via a confusion matrix. Finally, the confusion matrix is converted into percentage form.

7) Classification of the test cancer cell: To classify the test cancer cell, the system needs to pre-process the test image or test the cancer cell. For this purpose, the system has to apply pre-processing stages before applying classification. When image features are extracted using an activation function, then extracted image features passed to the classifier and the previously created classifier classifies the test image [1].

8) Predict class label: When the classifier classifies the test image, the classifier predicts a label that represents which class the test image belongs to. Based on this predicted class label, the next segmentation stage starts to process. This predicted class label helps the next process to minimize its dataset and its processing time or execution time also.

\section{B. Semantic Segmentation and Detection Stage}

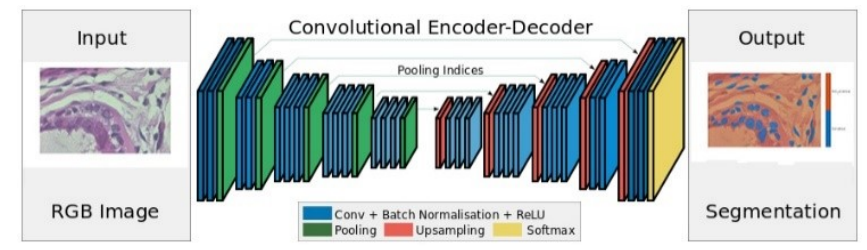

Fig. 4. Architecture of Semantic Segmentation.

"Fig. 4" illustrates the architecture of semantic segmentation. The encoders (i.e., a sequence of non-linear processing 
layers) are contained by the SegNet architecture. A pixel-wise classifier also follows the decoders (i.e., an equivalent group of decoders). Characteristically, one or more convolutional layers are contained by each encoder. Applying max-pooling directories in the encoding order, the decoder up-samples the light encoding because of the max-pooling [2].

To execute up-sampling of the low-resolution feature model in the decoders, max-pooling indices are used as one major component of the SegNet. This procedure bears some significant merits of holding the highest frequency niceties in segmented images. In the decoders, this procedure also helps to diminish trainable constraints. Using the stochastic gradient descent, the complete system can be accomplished end-to-end. The block diagram of semantic segmentation is given in "Fig. 5'.

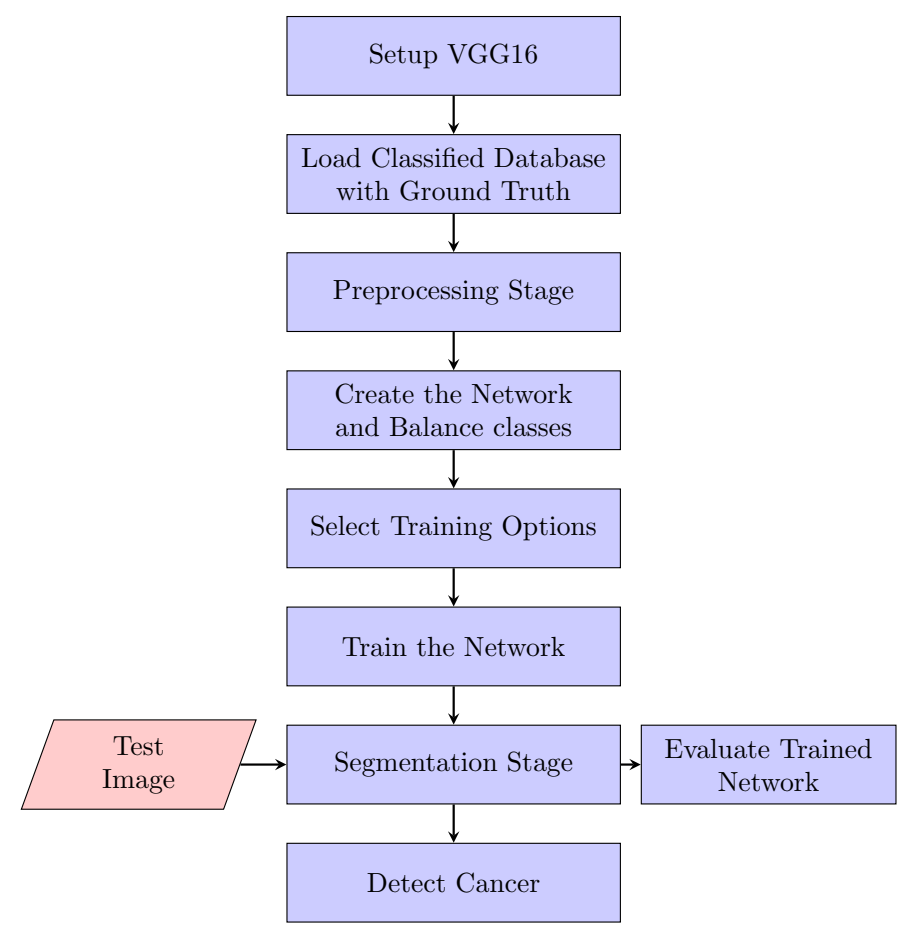

Fig. 5. Proposed block Diagram of Semantic Segmentation.

1) Setup VGG16: VGG-16 net is a 16 layers deep CNN net. The system loads a pre-trained network trained.To classify pictures into 1000 object classes (such as Brain, Breast, Leukemia, Lung and many other cancer types), the pretrained network is used. The system loaded a pretrained VGG-16 CNN net and examined the layers and categories. The pretrained VGG-16 network loaded using the vgg16 function. A sequence of the network object is used to produce output. The network has 41 layers. There are 16 layers with learnable weights: 3 fully connected layers and 13 convolutional layers exist in the net and all those 16 layers are learnable [3].

2) Load Classified Database with Ground Truth: After setting up the neural network, the system loaded the database with its ground truth. For this reason, the system classified the class of the test image. Based on the classified class the system loaded the only classified class database with its ground truth. Some labeled image with its original image is given in "Fig. 6".
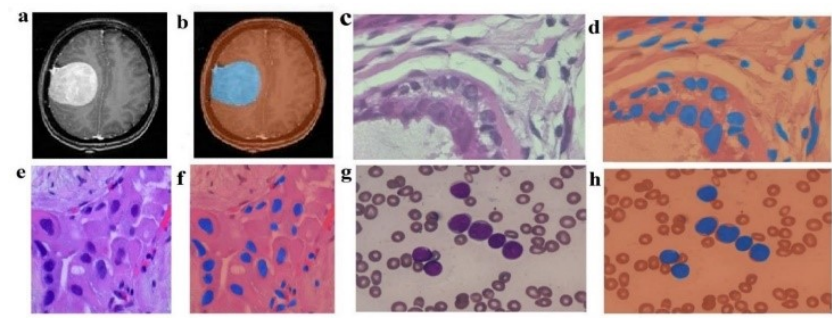

Fig. 6. Original images (a. Brain, c. Breast, e. Lung, g. Leukemia) and Labeling Images (b. Brain, d. Breast, f. Lung, h. Leukemia).

\section{3) Preprocessing Stage:}

a) Identify class and PixelLabelId: The system identified all class names and their corresponding pixel label id based on the classified loaded database. A large set of images and its counter set of labeled data are required to train a semantic segmentation network. To state the mapping between class names and label IDs, the pixel label data used. In the used dataset, the labels are "Cancerous" and "Non-cancerous" having 1, 2 label IDs respectively.

b) Analyze Dataset Statistics: In this study count pixels of Each Label used to observe the circulation of class labels in the database. The system can count the number of pixels in each label of a class and also can count the total pixels of each class in the used database. Using those pixel values, the percentage of cancer area and the non-cancer area are calculated. Those can be calculated using the following formula:

$$
\text { Frequency of each labels }=\frac{\text { Sum of label pixels }}{\text { Sum of image pixels }}
$$

c) Resize both Database Images and Labeled Images: In this research SegNet used the only process RGB images that are 300-by-300. To avoid re-saving all database images to this setup, the system used a converted and resized RGB image. The resized procedure has been applied not only for extra data augmentation, but also for training the network. Both the dataset image and labeled images are resized by the system.

d) Prepare both Training and Test Set: The system has described in the previous CNN section. The system needs to split the total dataset images and their pixel labels for training and testing sets. The system split the dataset $60 \%$ for the training set and $40 \%$ for the testing set.

4) Create the Network and Balance Classes: The system used the 'segnetLayers' procedure to make a neural network depending on VGG-16. The system specified the network image size. Then the number of categories is specified. Finally, the system created the SegNet layer network using image size, the number of categories and VGG-16. The classes are not balanced in an earlier dataset. To improve training, the system used class weight to equilibrium the classes. The pixel label computed earlier with a count each of label to compute the average frequency class weights, the pixel label measured past with the count of each label. The system identified class weights using a pixel classification layer. Then the system needs to update the pixel classification Layer of SegNet and delete the current pixel classification Layer and add the newly defined layer. 
5) Select Training Options: The gradient method used Stochastic gradient descent momentum (SGDM). The system also needs to adjust the mini-batch size according to the memory size installed in the GPU to be used. The system applied training options to regulate the hyper-constraints used for SGDM. A factor of 0.3 every 10 epochs has been used to diminish the learning rate. Setting the 'ValidationData' parameter against the validation data every epoch, the system has tested the network. When the validation accuracy joins, stopping the criteria of the training has been set to 4 by the 'ValidationPatience'. To diminish memory procedure while training, a mini-batch size of 8 has been used. Depending on the quantity of GPU on the system, this mini-batch size can increase or decrease. In addition, At the end of every training epoch, the saving of network checkpoints is enabled by this name-value pair. The system can continue training from the kept checkpoint, if training is hindered because of a system deduction or power outage.

6) Train the Network: Data augmentation has been used during training to progress the accuracy of the network. Data augmentation has been completed using randomized $\mathrm{X} / \mathrm{Y}$ translation and left/right reflection. The system used the image data augmenter to regulate these data augmentation constraints. Image data augmenter assists different other categories of data augmentation. During start training, the system combined data augmentation selections and the training data using pixel label image datastore. Batches of training data are read by the pixel label image datastore. To pass the resultant data of the augmentation process to the training process, data augmentation is applied. Pixel label image source is used to define the final data used for learning. Augmentation is also done here. After completing training, the system can segment the test image of the cancer cell using the created semantic segmentation network. For this reason, the system has to load the test image to the semantic segmentation network.

7) Semantic Segmentation: The field of computer vision has been empowered by deep learning. Semantic segmentation is one part of computer vision. Labeling each pixel is the purpose of semantic segmentation. Because the system is predicting for each pixel in the image, the dense prediction is referred to as the system. As the system created the SegNet network and trained it successfully, the system can now segment the loaded test image to the network. Using the trained net, the system can segment the test image and overlap the pixel label in it. It can be seen that the regions have been relatively neatly divided. Some test images are given in "Fig. 7".
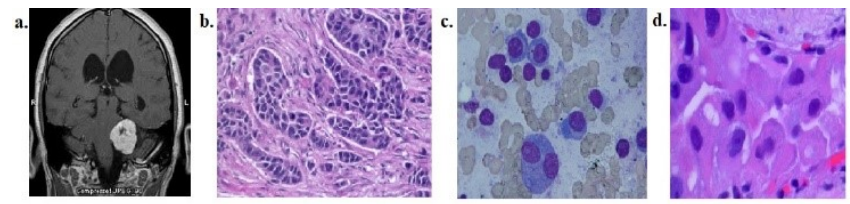

Fig. 7. Input Images (a. Brain, b. Breast, c. Leukeima, d. Lung).

The system can compare the segmentation result with the ground truth. As a result of overwriting, the green and magenta areas are different from the true values. For each class segmented by SegNet, the system can evaluate how much the ground truth region is included. This is an index called $\mathrm{IoU}$ and can be measured using the jaccard function. In addition to jaccard, the dice coefficient and BF coefficient are often used as the coefficient for expressing the similarity to the true value. The system uses the dice function and the bfscore function on MATLAB respectively. Segmented result of the test images given in "Fig. 8".
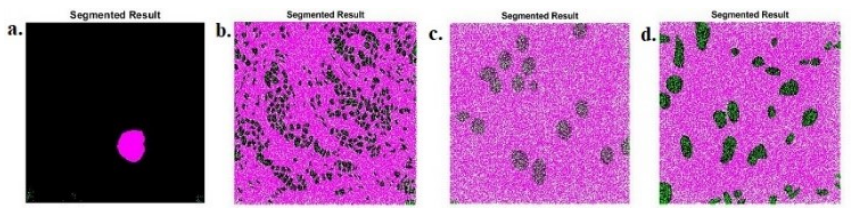

Fig. 8. Segmented Results (a. Brain, b. Breast, c. Leukemia, d. Lung).

8) Evaluate Trained Network: Semanticseg function has been run on the complete test set, to calculate the accuracy. Semanticseg returns the outcomes as a pixel label datastore object. The 'WriteLocation' parameter is used to write the location of the actual pixel label data. The system used the evaluate semantic segmentation function to calculate semantic segmentation metrics. For individual classes and for each input image, the semantic segmentation process returns various metrics for the complete dataset. The dataset metrics used to identify the network performance.

9) Detect Cancer: After segmentation, the system can detect any object from the segmentation output. Based on the cancer cell labeled, the system can separate the cancer cell from another labeling object. To get a meaningful segmentation output, the system applies different morphological operations and finally converts it into a binary form so that it can separate the cancer cell and others. Cancer cells represented in white e.g. 1 and non-cancerous are represented as black e.g. 0 [15]. The binary representation of segmented cancer cells given in "Fig. 9".
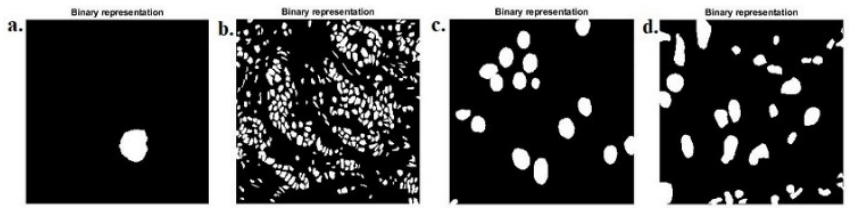

Fig. 9. Binary Representation (a. Brain, b. Breast, c. Leukemia, d. Lung).

After detecting cancer cells, the boundary of the cancer cells is identified by applying boundary detection algorithms and the boundary is represented by the red color. Detected cancer output is given in "Fig. 10".
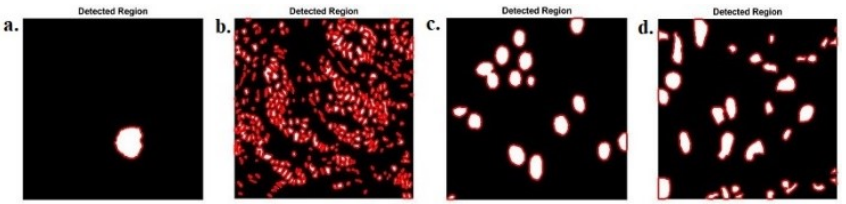

Fig. 10. Detected Region (a. Brain, b. Breast, c. Leukemia, d. Lung). 


\section{Area Calculation}

Traced and detected cancerous outcomes are in the binary form. So, to calculate the percentage area of the cancerous region, the intensity (i.e., 1) of each pixel is summed and the total number of pixels of the test image is counted. The following equation is used to count both the area of the detected cancerous area and the total area of the test image [3].

$$
\text { Area }=\sum_{i=1, j=1}^{n, m} P(i, j)
$$

In the system, the cancerous area is identified with the pixel intensity having ones and the non-cancerous area identified with the pixel intensity zeros [1]. The percentage of the cancerous area is calculated with the ratio of the cancerous area and the total test image are as follows:

The percentage area $=\frac{\text { The area of Cancerous Cell }}{\text { The area of tested image }} \times 100 \%$

\section{RESUlt AND Discussion}

This paper developed a system to experiment on different databases of different types of cancerous cells. For brain cancer or brain tumor dataset purpose, the authors used Brain MRI Images for Brain Tumor Detection dataset. It consists of 154 brain images. For breast cancer histopathological annotation and diagnosis (BreCaHAD) dataset contains required files for Breast Cancer Histopathological Annotation and Diagnosis. It contains three folders named groundTruth_display (ground truth used on actual images), groundTruth (json files), images (original images). From those folders, the authors used only original images. This dataset contains 162 test images. For leukemia, the authors used SN-AM Dataset [16][17][18][19][20], which has three subsets. They used subset-2, and subset-3 where each of the subset contained 30 images. Lung and Colon Cancer Histopathological Images dataset [21], which consists of 25000 histopathological images and five categories, is used for Lung Cancer. Each image's size is $768 \times 768$ pixels, and each of them is in a jpeg file format. The authors created new polygonal ground truth for all datasets.

For evaluation of the proposed methodology Intersection over Union (IoU) is used. To evaluate IoU, detected cancerous areas and another area counted from ground truth are required. More formally, the hand-labeled ground truth that can form a polygon in the cancerous region and another polygon created with detection from the system methodology is required to estimate the proposed system accuracy using IoU. As the system used by the hand labeled ground-truth of different datasets and applied Intersection over Union (IoU) evaluation metric [22].

$$
I o U=\frac{\text { Region of the Overlap }}{\text { Region of the Union }}
$$

Table I. illustrates the IoU measuring raw values calculated from the total database and individual IoU. Calculating the
TABLE I. EVALUATION OF THE SYSTEM

\begin{tabular}{|c|c|c|c|}
\hline Cancer Type & Area of Overlap & Area of Union & IoU \\
\hline Brain & 93,195 & 94,529 & 0.9859 \\
\hline Breast & $2,724,105$ & $2,988,369$ & 0.9116 \\
\hline Leukemia & 597,690 & 642,560 & 0.9302 \\
\hline Lung & $72,695,210$ & $80,560,420$ & 0.9024 \\
\hline
\end{tabular}

average IoU from the above table, the system produces $93 \%$ accuracy.

Again, in the classification, the confusion matrix is applied to evaluate the exactness of CNN. It is used to identify the accuracy of the classifier. By observing how the classifier predicts a correct one as a truly positive and also observing a relationship of each category it can predict a class. The main job of a confusion matrix is to predict the results of a classifier and identify how correctly the classifier predicts a known class. Using true negative (x), true positive (w), false negative $(\mathrm{z})$ and false positive (y) of the confusion matrix, the system can calculate the following performance.

$$
\begin{gathered}
\text { Accuracy }(\text { Ac })=\frac{w+x}{w+x+y+z} \times 100 \\
\text { Misclassification Rate }=1-\text { Accuracy } \\
\text { Specificity }=\frac{x}{x+y} \times 100 \\
\text { Precision }(\text { Ps })=\frac{w}{w+y} \times 100 \\
\text { Recall }(\text { Re })=\frac{w}{w+z} \times 100 \\
\text { F- measure }(F-m)=\frac{2 \times \text { Recall } \times \text { Precision }}{\text { Recall }+ \text { Precision }} \times 100
\end{gathered}
$$

TABLE II. Performance CAlculation of CNN Classifier

\begin{tabular}{|c|c|c|c|c|c|c|c|c|}
\hline Cancer Type & $\mathbf{w}$ & $\mathbf{x}$ & $\mathbf{y}$ & $\mathbf{z}$ & $\mathbf{A c}(\boldsymbol{\%})$ & $\mathbf{P s}(\boldsymbol{\%})$ & $\mathbf{R e}(\boldsymbol{\%})$ & $\mathbf{F}-\mathbf{m}(\boldsymbol{\%})$ \\
\hline Brain & 18 & 54 & 0 & 0 & 100 & 100 & 100 & 100 \\
\hline Breast & 18 & 54 & 0 & 0 & 100 & 100 & 100 & 100 \\
\hline Leukemia & 18 & 54 & 0 & 0 & 100 & 100 & 100 & 100 \\
\hline Lung & 18 & 54 & 0 & 0 & 100 & 100 & 100 & 100 \\
\hline
\end{tabular}

Table II shows that the CNN classifier gave a $100 \%$ accuracy rate.

\section{CONCLUSiON}

This research overcame the limitation of using individual methods for the cancer detection system for both MRI and Histopathology images. The results obtained in this research notify that the author's strategy is practical and can also pointedly support precise, can automatically detect cancerous cells and the proposed combination gives better accuracy after applying the machine learning algorithm. The authors reduced 
the training time and increased the segmentation accuracy using a CNN classifier and SegNet model with a morphological operation which is better than the common SegNet architecture. The experimental result achieves detection of cancer cells from various cancer databases that represent proper accuracy rates of an average of $93 \%$.

Future research will be focused on using the more accurate, advanced deep learning algorithms to reduce the time complexity for large datasets and improve the accuracy rate. We will use more types of cancer cells and their categories. This research will open many opportunities for further research in image segmentation and cancer cell detection.

\section{REFERENCES}

[1] Sikder, Juel, Utpol Kanti Das, and AM Shahed Anwar. "Cancer Cell Segmentation Based on Unsupervised Clustering and Deep Learning." In International Conference on Intelligent Computing \& Optimization, pp. 607-620. Springer, Cham, 2020.

[2] Lateef, Fahad, and Yassine Ruichek. "Survey on semantic segmentation using deep learning techniques." Neurocomputing 338 (2019): 321-348.

[3] Alqazzaz, Salma, Xianfang Sun, Xin Yang, and Len Nokes. "Automated brain tumor segmentation on multi-modal MR image using SegNet." Computational Visual Media 5, no. 2 (2019): 209-219.

[4] Ghoneim, Ahmed, Ghulam Muhammad, and M. Shamim Hossain. "Cervical cancer classification using convolutional neural networks and extreme learning machines." Future Generation Computer Systems 102 (2020): 643-649.

[5] Kamnitsas, Konstantinos, Christian Ledig, Virginia FJ Newcombe, Joanna P. Simpson, Andrew D. Kane, David K. Menon, Daniel Rueckert, and Ben Glocker. "Efficient multi-scale 3D CNN with fully connected CRF for accurate brain lesion segmentation." Medical image analysis 36 (2017): 61-78.

[6] Almajalid, Rania, Juan Shan, Yaodong Du, and Ming Zhang. "Development of a deep-learning-based method for breast ultrasound image segmentation.” In 2018 17th IEEE International Conference on Machine Learning and Applications (ICMLA), pp. 1103-1108. IEEE, 2018.

[7] Al-jaboriy, Saif S., Nilam Nur Amir Sjarif, Suriayati Chuprat, and Wafaa Mustafa Abduallah. "Acute lymphoblastic leukemia segmentation using local pixel information." Pattern Recognition Letters 125 (2019): 85-90.

[8] Kamal, Uday, Abdul Muntakim Rafi, Rakibul Hoque, Jonathan Wu, and Md Kamrul Hasan. "Lung cancer tumor region segmentation using recurrent 3d-denseunet." In International Workshop on Thoracic Image Analysis, pp. 36-47. Springer, Cham, 2020.

[9] Mahmud, Tanjim, Juel Sikder, Rana Jyoti Chakma, and Jannat Fardoush. "Fabric Defect Detection System." In International Conference on Intelligent Computing \& Optimization, pp. 788-800. Springer, Cham, 2020.

[10] Tanjim Mahmud, Juel Sikder, Umme Salma, Sultana Rokeya Naher, Jannat Fardoush, Na-hed Sharmen, Sajib Tripura. "An Optimal Learning Model for Training Expert System to Detect Uterine Cancer" The 12th International Conference on Ambient Systems, Networks and
Technologies (ANT) March 23-26, 2021, Warsaw, Poland, Procedia Computer Science 184 (2021) 356-363. Published by Elsevier B.V, https://doi.org/10.1016/j.procs.2021.03.045.

[11] de Brebisson, Alexander, and Giovanni Montana. "Deep neural networks for anatomical brain segmentation." In Proceedings of the IEEE conference on computer vision and pattern recognition workshops, pp. 20-28. 2015.

[12] Damodharan, Selvaraj, and Dhanasekaran Raghavan. "Combining tissue segmentation and neural network for brain tumor detection." International Arab Journal of Information Technology (IAJIT) 12, no. 1 (2015).

[13] Wang, Meng, Xiaobo Zhou, Fuhai Li, Jeremy Huckins, Randy W. King, and Stephen TC Wong. "Novel cell segmentation and online learning algorithms for cell phase identification in automated time-lapse microscopy." In 2007 4th IEEE International Symposium on Biomedical Imaging: From Nano to Macro, pp. 65-68. IEEE, 2007.

[14] Tanjim Mahmud, Sajib Tripura, Umme Salma, Jannat Fardoush, Sultana Rokeya Naher, Juel Sikder and Md Faisal Bin Abdul Aziz. "Face Detection and Recognition System", 2nd In-ternational Conference on Technology Innovation and Data Sciences (ICTIDS)-2021, Kuala Lumpur, Malaysia, Published in Lecture Notes in Networks and Systems" Series (LNNS) - Springer Nature. DOI: 10.1007/978-981-16-3153-5.

[15] Alexandre de Brebisson, Giovanni Montana, "Deep neural networks for anatomical brain segmentation", In Proceedings of the IEEE Conference on Computer Vision and Pattern Recognition Workshops, pp. 20-28, 2015.

[16] Gupta, Anubha, Rahul Duggal, Shiv Gehlot, Ritu Gupta, Anvit Mangal, Lalit Kumar, Nisarg Thakkar, and Devprakash Satpathy. "GCTI-SN: Geometry-inspired chemical and tissue invariant stain normalization of microscopic medical images." Medical Image Analysis 65 (2020): 101788.

[17] Gupta, Ritu, Pramit Mallick, Rahul Duggal, Anubha Gupta, and Ojaswa Sharma. "Stain color normalization and segmentation of plasma cells in microscopic images as a prelude to development of computer assisted automated disease diagnostic tool in multiple myeloma." Clinical Lymphoma, Myeloma and Leukemia 17, no. 1 (2017): e99.

[18] Duggal, Rahul, Anubha Gupta, Ritu Gupta, Manya Wadhwa, and Chirag Ahuja. "Overlapping cell nuclei segmentation in microscopic images using deep belief networks." In Proceedings of the Tenth Indian Conference on Computer Vision, Graphics and Image Processing, pp. 1-8. 2016.

[19] Duggal, Rahul, Anubha Gupta, and Ritu Gupta. "Segmentation of overlapping/touching white blood cell nuclei using artificial neural networks." CME Series on Hemato-Oncopathology, All India Institute of Medical Sciences (AIIMS), New Delhi, India (2016).

[20] Duggal, Rahul, Anubha Gupta, Ritu Gupta, and Pramit Mallick. "SDlayer: stain deconvolutional layer for CNNs in medical microscopic imaging." In International Conference on Medical Image Computing and Computer-Assisted Intervention, pp. 435-443. Springer, Cham, 2017.

[21] Borkowski AA, Bui MM, Thomas LB, Wilson CP, DeLand LA, Mastorides SM. Lung and Colon Cancer Histopathological Image Dataset (LC25000). arXiv:1912.12142v1 [eess.IV], 2019.

[22] Zheng, Zhaohui, et al. "Distance-IoU loss: Faster and better learning for bounding box regression." Proceedings of the AAAI Conference on Artificial Intelligence. Vol. 34. No. 07. 2020. 